\title{
NeuroRegulation
}

\section{Neurofeedback Practitioner Factors Related to Client Adherence}

\author{
Jonathon E. Larson*, Lindsay L. Sheehan. Thomas P. Cothran, Kelly O'Neill, and \\ Bethany Apa \\ Illinois Institute of Technology, Chicago, Illinois, USA \\ *Address correspondence to: Jonathon Larson, Illinois Institute of Technology, 3424 S. State, First Floor, Room 1B9-2, \\ Chicago, IL 60616. Email: larsonjon@iit.edu \\ Copyright: (c) 2014 Larson et al. This is an Open Access article distributed under the terms of the Creative Commons \\ Attribution License (CC-BY).
}

\begin{abstract}
Introduction. This study systematically identified, extracted, and organized neurofeedback (NFB) practitioner factors connected to client adherence. It is important to understand this connection because increased adherence leads to improved NFB outcomes. A previous NFB conceptual framework and previous NFB client adherence findings were used to guide the current study.

Method. One hundred and ninety-eight NFB practitioners completed online surveys gathering demographic information and ratings of practice behaviors and characteristics. For data set analyses, this study utilized SPSS version 20 for descriptive statistics, frequencies, means, standard deviations, ranges, Pearson product-moment correlation analyses, and independent samples $t$-tests.

Results. Findings indicated that the following significantly correlated with client adherence: (a) practitioner technical and interpersonal techniques; (b) practitioner commitment to improving technical and interpersonal skills; and (c) practitioner confidence displayed during sessions. Results also indicated commitment correlated separately with techniques and confidence. These results suggested that practitioners engaging in self-NFB sessions reported significantly higher adherence rates compared to practitioners not engaging in selfNFB sessions. Findings demonstrated that practitioners conducting $\geqq 40$ monthly NFB sessions reported significantly higher adherence rates compared to practitioners conducting $<40$ monthly NFB sessions.

Conclusion. This study concluded that practitioners with commitment to improving their technical and interpersonal expertise leads to increased confidence during NFB sessions, ultimately improving adherence and outcome rates. When averaging 40 or more NFB sessions with clients per month, practitioners provide themselves with continued opportunities to practice current and new technical and interpersonal skills. By conducting self-NFB, practitioners develop their own descriptions of physiological regulation and share their own results with clients, which in turn builds rapport and increases therapeutic bonds leading to higher adherence.
\end{abstract}

Keywords: Practitioners, neurofeedback, EEG biofeedback, brain-computer interface, adherence 


\section{Introduction}

Neurofeedback, electroencephalographic (EEG) biofeedback, or brain-computer interface merges advanced technology and operant conditioning to teach individuals to influence their EEG patterns leading to improved physiological regulation and psychological functioning. For the purposes of this paper, the term neurofeedback (NFB) was utilized; however, this term also refers to electroencephalographic biofeedback and brain-computer interface. These terms were also utilized in the literature reviews for this paper.

Research has demonstrated the positive effects of NFB on various physiological and psychological disorders. Arns, de Ridder, Strehl, Breteler, and Coenen's (2009) neurofeedback meta-analysis reported large effect sizes for impulsivity and inattention and a medium effect size for hyperactivity. A randomized controlled trial with a six-month follow-up of children with ADHD indicated significant academic improvements for the NFB intervention group compared to the pharmacological intervention group (Meisel, Servera, Garcia-Banda, Cardo, \& Moreno, 2013). Niv (2013) reviewed NFB effectiveness research for various disorders and concluded that NFB demonstrated superior or equivalent outcomes when compared to alternative or no treatment. To organize continued advancement of NFB research, Yucha and Montgomery (2008) published an evidence-based framework, and Hammond (2011) provided an extensive review of NFB research findings.

In addition to NFB efficacy and effectiveness research, current literature highlights the importance of exploring practitioner and client relationships, establishing NFB practice guidelines, identifying properly trained practitioners, highlighting NFB learning principles, and understanding potential directions for future practice and research growth (Aguilar-Prinsloo \& Lyle, 2010; Hammond \& Kirk, 2008; Hammond et al., 2011; Sherlin et al., 2011; Lyle, 2012). This study pursued these recommendations through investigating practitioner factors related to client adherence within NFB settings. A crucial aspect of NFB feasibility and effectiveness research included exploring client adherence since discontinuation of recommended NFB treatment plans negatively affects physiological and psychological outcomes. The World Health Organization (2003) defined adherence as client behaviors that correspond with a collaborative plan of action developed with health care practitioners. Current adherence literature demonstrates that $20-30 \%$ of clients do not fill their first medication prescription or attend their first therapy appointment, $50 \%$ of clients drop out of behavioral and medication treatments, and $25-50 \%$ drop out of services during the first year of treatment (World Health Organization, 2003; Fischer et al., 2011). Specifically, previous research indicated client adherence problems exist within NFB settings with adherence connected to practitioner quality of work life, frequency of NFB sessions, practitioner NFB knowledge levels, commitment to practice improvement, and mentorship (Larson, Ryan, \& Baerentzen, 2010; Larson, Cothran, Drandorff, Morgan, \& Ryan, 2012).

Based on previous mental health practitioner literature (Grencavage \& Norcross, 1990; Larson, Ryan, \& Baerentzen, 2010; Larson, Cothran, Drandorff, Morgan, \& Ryan, 2012; Tracey, Lichtenberg, Goodyear, Claiborn, \& Wampold, 2003; Wampold, Mondin, Moody, Benson, \& Ahn, 1997), this study explored connections between client adherence and practitioner variables including: NFB techniques; commitment; empathy, confidence, friendliness, optimism, monthly NFB sessions, and self-NFB sessions. First, variables utilized in this study are provided, and then specific measurement details of each variable are described in the methods section of this paper. Second, a current literature review and a rationale for including these variables in this study are provided. Third, the study hypotheses are offered. 


\section{Definitions of Variables}

Throughout this section of the paper, the primary variables are typed in bold to provide easy reference for the reader. The World Health Organization (2003) defined client adherence as client behaviors corresponding with a collaborative plan of action developed with health care practitioners. This study defined techniques as practitioner abilities utilizing both NFB technology and interpersonal skills. This paper identified commitment as the level of importance practitioners place on learning new NFB technology and interpersonal skills. Empathy included the ability to display active understanding of a client's situation, and confidence described self assurance in providing effective therapeutic treatment during NFB sessions. This paper defined friendliness as providing comfortable and engaging conversations during sessions and optimism as maintaining a positive outlook throughout the therapeutic process. Monthly sessions included the total number of NFB sessions that practitioners provided each month. Self-NFB sessions included the total number of NFB sessions that practitioners apply to themselves each month.

\section{Study Rationale}

Client adherence literature demonstrates alarming rates of failure to attend first appointments and high dropout rates for both behavioral and medication treatments; however, increasing adherence rates improves health and psychological outcomes (World Health Organization, 2003; Fischer, 2011). Previous research indicated that client adherence problems exist within NFB settings (Larson, Ryan, \& Baerentzen, 2010); with this evidence of client adherence problems, the current study proposes that is important to continue adherence research within NFB in order to improve health outcomes. Previous research connected client adherence to frequency of monthly NFB sessions, NFB techniques, and commitment to practice improvement (Larson, Cothran, Drandorff, Morgan, \& Ryan, 2012). Substantiating previous research and building understanding of NFB adherence, this paper proposes to investigate these variables. This paper postulates that self-NFB is related to adherence because practitioners who use NFB themselves are able to develop their own descriptions of physiological and psychological regulation. Doing self-NFB also allows practitioners to engage in self-disclosure about similar NFB experiences and outcomes. By sharing their own results with clients, practitioners build rapport and improve the therapeutic bond leading to improved adherence rates.

This study also investigates empathic, confident, friendly, and optimistic qualities because practitioners reported the importance of these traits within NFB settings in previous studies (Larson, Ryan, \& Baerentzen, 2010; Larson, Cothran, Drandorff, Morgan, \& Ryan, 2012). Other research also identified these items as important therapist qualities (Grencavage \& Norcross, 1990; Wogan, \& Norcross, 1985). Imel and Wampold's (2008) psychotherapy common factors framework organized the four NFB practitioner characteristics of empathic, confident, friendly, and optimistic. Imel and Wampold defined common factors as practitioner characteristics, role, client bond, context, and relationship qualities, which are separate from the specific therapy method being applied. A meta-analysis reported that up to $70 \%$ of client outcomes can be explained by common factors rather than method of therapy (Wampold, Mondin, Moody, Stich, Benson, \& Ahn, 1997). Since their framework includes a broad range of factors and this study was only focused on practitioner factors, this study modified the common factors model into a common NFB practitioner factors model that included four practitioner factors. This study offers the following hypotheses based on previous literature and rationales. 


\section{Research Hypotheses}

1. Empathic, confident, friendly, and optimistic scores will be separately correlated with adherence scores.

2. Adherence will be separately correlated with techniques, commitment, and confidence.

3. The group with high rates of monthly sessions will report higher adherence rates compared to the group with low rates of monthly sessions.

4. The group that completes self-NFB sessions will report higher adherence rates compared to the group that does not complete self-NFB sessions.

\section{Method}

\section{Participants and Procedure}

With Illinois Institute of Technology institutional review board approval, the study team recruited NFB practitioners through discussion boards and email distribution. The announcement directed participants to an online survey that included a consent process. This study collected 198 usable practitioner surveys and utilized SPSS Version 20.0 to complete study analyses. Two research assistants entered the surveys into two separate SPSS files; discrepancies were resolved by comparing the two files and original surveys. A five-step data set cleaning process was utilized to identify errors, missing data, and outliers, and to ensure data met assumptions for the analyses (Mickey, Dunn, \& Clark, 2004). Descriptive statistics, frequencies, means, standard deviations, ranges, Pearson productmoment correlation analyses, and independent samples $t$-tests were calculated for SPSS data set analyses.

\section{Instrumentation}

This study collected responses to the 65-item NFB Practitioner Survey, which can be found in Appendix A. This survey was developed by utilizing findings from previous NFB practitioner investigations (Larson, Ryan, \& Baerentzen, 2010; Larson, Cothran, Drandorff, Morgan, \& Ryan, 2012; Larson, In Press). This survey included demographic information and ratings on practitioner characteristics. The variables from the 65 -item survey that were used for the remaining analyses are described below. The following variables utilized one survey question: gender (item \#1), age (item \#2), education (item \#3), mental health license (item \#4), health care license (item \#5), experience (item \#6), continuing education (item \#7), monthly NFB sessions (item \#8), and self-NFB (item \#17). The following variables utilized two or more survey questions. Client adherence was calculated by subtracting monthly dropouts (item \#10) from successful monthly closures (item \#9). This study measured techniques by adding the scores of two survey questions: "How would you rate your current knowledge about neurofeedback technology?" (item \#11) and "How would you rate your interpersonal skills with clients?" (item \#12). Both were measured on a 7-point Likert scale with the anchors of " $1=$ poor" to " $7=$ excellent". These questions gathered practitioner perspectives of their own knowledge levels rather than testing their knowledge or obtaining someone else's rating of their knowledge. Commitment was measured by adding the scores of two survey questions: "How would you rate your commitment to learning about neurofeedback technology?" (item \#13) and "How would you rate your commitment to improving interpersonal skills with clients?" (item \#14). Both were measured on a 7-point Likert scale with the anchors of " $1=$ poor" to " 7 = excellent". 
Using 7-point Likert scales, ability, priority, ease, and frequency were measured for: empathic, confident, friendly, and optimistic. For example, "During a neurofeedback session, what is your satisfaction level with your ability to be confident?" $(1$ = very dissatisfied to $7=$ very satisfied); "During a neurofeedback session, what is your priority level for being confident?" ( 1 = not a priority to 7 = essential priority); "During a neurofeedback session, what is your level of difficulty or ease with being confident?" ( $1=$ very difficult to $7=$ very easy); "During a neurofeedback session, how often are you confident?" ( $1=$ not at all to $7=$ frequently). The same method of measurement was used for the remaining three factors of empathic, friendly, and optimistic. This study added the four scores from each question to obtain a composite factor score. For example, the composite confident score = confident ability score + confident priority score + confident ease score + confident frequency score. Composite scores for empathic, confident, friendly, and optimistic factors were used for the remaining analyses of this study.

\section{Results}

Table 1 presents demographic information for research subjects utilized in this study. For 198 subjects, percentages for gender, education, mental health licensure, and healthcare licensure were provided; in addition, means and standard deviations were provided for age, years practicing NFB, number of NFB sessions monthly, and continuing education.

Table 2 provides means, standard deviations, and ranges for variables utilized in the remaining analyses. The variables included: adherence, techniques, commitment, and confidence. These results were used for the Pearson product-moment correlation analyses.

Table 3 provides Pearson product-moment correlations for adherence, techniques, commitment, and confidence. Results indicated significant correlations between variables of interest in this study, and implications are discussed within the conclusion section.

An independent samples $t$-test was conducted to compare adherence in the no self-NFB condition and the self-NFB condition. There was a significant difference in the scores for no self-NFB $(M=4.01, S D=9.21)$ and self-NFB $(M=7.89, S D=16.28)$ conditions, $t(196)=-$ 2.09, $p=0.038$. These results suggest that self-NFB affects client adherence; specifically, results suggest that when practitioners engage in self-NFB, their clients' adherence increases. An independent samples $t$-test was conducted to compare adherence in the fewer than 40 monthly NFB sessions condition and the $\geqq 40$ monthly sessions condition. There was a significant difference in the scores for the $<40$ monthly sessions $(M=3.08, S D=$ $10.60)$ and the $\geqq 40$ monthly sessions ( $M=6.62$, $S D=12.12$ ) conditions, $t(196)=-2.16, p=$ 0.032 . These results suggest that frequency of monthly sessions affects client adherence; specifically, results suggest that when practitioners conduct $\geqq 40$ monthly sessions, their client adherence increases. 


\section{Table 1}

Demographic Information for Neurofeedback Practitioners ( $N=198)$

\begin{tabular}{|c|c|c|c|}
\hline Item & $M$ & $S D$ & $\%$ \\
\hline \multicolumn{4}{|l|}{ Gender } \\
\hline Female & -- & -- & 48.00 \\
\hline Male & -- & -- & $\underline{52.00}$ \\
\hline Total & -- & -- & 100.00 \\
\hline \multicolumn{4}{|l|}{ Education } \\
\hline Associates & -- & -- & 1.00 \\
\hline Bachelors & -- & -- & 7.60 \\
\hline Masters -- & & -- & 39.90 \\
\hline Doctorate & -- & -- & 51.50 \\
\hline Total & -- & -- & 100.00 \\
\hline \multicolumn{4}{|c|}{ Mental Health Licensure } \\
\hline License -- & & -- & 76.30 \\
\hline Non-License & -- & -- & 23.70 \\
\hline Total & -- & -- & 100.00 \\
\hline \multicolumn{4}{|l|}{ Healthcare Licensure } \\
\hline License -- & & -- & 69.20 \\
\hline Non-License & -- & -- & $\underline{30.80}$ \\
\hline Total & -- & -- & $\overline{100.00}$ \\
\hline Age & 55.70 & 11.19 & -- \\
\hline Years Practicing NFB & 9.96 & 7.61 & -- \\
\hline Monthly Sessions & 62.45 & 69.82 & -- \\
\hline \multicolumn{4}{|l|}{ Monthly Continuing } \\
\hline Education & 6.00 & 7.38 & -- \\
\hline
\end{tabular}




\section{NeuroRegulation}

Table 2

Means, Standard Deviations, and Range of Adherence, Techniques, Commitment, Confidence, Monthly Sessions, and Self-NFB Scores $(N=198)$

\begin{tabular}{llcl}
\hline Measure & $M$ & $S D$ & \multicolumn{1}{l}{ Range } \\
Adherence & 5.03 & 11.57 & $-5.00-95.67$ \\
Techniques & 11.31 & 1.57 & $7.00-14.00$ \\
Commitment & 12.04 & 1.81 & $7.00-14.00$ \\
Confidence & 23.79 & 3.11 & $14.00-28.00$ \\
Monthly & & & $0.00-400.00$ \\
Sessions & 62.45 & 69.82 & $1.00-6.00$ \\
Self-NFB & 2.6 & 1.41 & \\
\hline
\end{tabular}

Table 3

Findings from Correlations of NFB Practitioners' Adherence, Techniques, Commitment, and Confidence Scores $(N=198)$

Scale $\quad$ A $\quad T \quad$ Com Con

A $\quad--\quad .15^{*} \quad .16^{*} \quad .18^{*}$

T $\quad--\quad--\quad .55^{\star *} \quad .43^{\star *}$

$\begin{array}{lllll}\text { Com } & -- & -- & -- & .46^{\star \star}\end{array}$

Note. $\mathrm{A}=$ Adherence, $\mathrm{T}=$ Techniques, Com $=$ Commitment, Con $=$ Confidence, ${ }^{*} p<.05,{ }^{* *} p$ $<.01$. 


\section{Discussion}

The first hypothesis was partially supported by the Pearson product-moment correlation analysis findings; a significant correlation between confidence and adherence was found. Clients may be less likely to drop out and be more likely to complete training recommendations when practitioners engage with confidence about applying NFB training sessions, planning a course of treatment, and describing outcomes. Practitioners that display high levels of confidence during NFB sessions provide clients with reassurance, which in turn increases the likelihood of attending future sessions. Practitioners who model confidence also promote therapeutic relationships that augment recommended NFB treatment goals. Activities that may contribute to increasing practitioner confidence include: attending NFB workshops, utilizing mentorship opportunities, completing NFB certifications, increasing NFB technology knowledge, and increasing interpersonal skills. This study did not find significant relationships between client adherence and empathic, friendly, and optimistic traits. Potentially, these three factors do not influence client decisions about continuing NFB sessions. It is also possible that the current study design, measurement methods, and analyses may be limited in measuring and identifying empathic, friendly, and optimistic traits as factors related to adherence. Further research on adherence may include surveying clients on practitioner factors that promote treatment adherence.

The second hypothesis was supported by the correlation analysis findings; significant and separate correlations between adherence and techniques, commitment, and confidence were found. In addition, significant and separate correlations between commitment, techniques, and confidence were indicated. Commitment to improving NFB techniques and interpersonal skills increases adherence rates leading to higher rates of positive outcomes. Commitment also increases confidence in practice skills that lead to improved adherence rates. Practitioners displaying confidence and commitment during sessions may influence clients to increase their own commitment to and confidence about the NFB process. When clients expect and experience positive outcomes, treatment adherence and willingness to complete NFB therapy goals increases.

The third hypothesis was supported by an independent samples $t$-test. Group one with $\geqq 40$ monthly sessions reported significantly higher adherence rates compared to group two with $<40$ monthly sessions. Results suggested that the frequency of monthly sessions affects client adherence. It is possible that increasing the number of monthly sessions provides more opportunities to improve NFB application skills, which in turns produces successful outcomes leading to higher client adherence. To improve client adherence, practitioners may focus on strategies that increase the time available to complete NFB sessions. One strategy may include hiring/contracting personnel to coordinate scheduling, billing, marketing, and other administrative tasks that take time away from running sessions.

The fourth hypothesis was supported by an independent samples $t$-test; there was a significant difference in the scores for the no self-NFB sessions group versus the self-NFB sessions group. Results suggested that practitioners completing self-NFB reported higher adherence rates. When practitioners practice self-NFB they are able to develop their own descriptions of physiological and psychological regulation, which in turn, allows them to use these descriptions to discuss the NFB process with clients. While self disclosing NFB experiences and outcomes, practitioners build rapport and improve the therapeutic bond leading to higher adherence rates. 
A wealth of robust research has indicated positive results of NFB therapy on client outcomes; however, this paper also emphasized the importance of exploring the influence of practitioner factors on adherence. Client adherence can be influenced by practitioners that display confidence during sessions, average 10 or more sessions per week, practice self-NFB, and maintain a commitment to improving techniques and interpersonal skills. These study findings offer guidance for future adherence research and for understanding adherence from a practitioner's viewpoint. Incorporating these findings within mentorship contacts, NFB workshops, and/or university courses may improve awareness of factors influencing adherence. Providing brief reviews about NFB adherence within educational settings may initiate discussions about problems and potential adherence strategies among new or experienced practitioners. Mentors, trainers, and teachers providing opportunities to discuss adherence problems prepare practitioners to incorporate adherence solutions within their practice. Future NFB client adherence research may include testing the feasibility and the impact of incorporating adherence components within NFB sessions. Comparing NFB education sessions with and without adherence training components may produce fruitful insights connected to improving NFB adherence outcomes. Future research may focus on exploring client perceptions of practitioner levels of commitment, techniques, and confidence within NFB sessions, since exploring client viewpoints of adherence may also improve an understanding of process and outcome factors. With these findings, the development of a NFB practitioner common-factors framework to organize practitioner factors may improve efficiency for future adherence and outcomes research.

These findings are not a comprehensive list of variables that influence adherence. This study collected practitioner self-perceptions and did not collect client data; this leads to limitations in generalization and ability to connect practitioner self-perceptions with client adherence. Additional factors may have been missed due to the study design, sample size, and method of data collection. Overall, this study attempted to identify practitioner self-perceptions connected to adherence for future NFB research. 


\section{References}

Aguilar, S., \& Lyle, R. (2010). Client perception of the neurofeedback experience: The untold perspective. Journal of Neurotherapy, 14, 55-60. http://dx.doi.org/10.1080/10874200903543948

Arns, M., de Ridder, S., Strehl, U., Breteler, M., \& Coenen, A. (2009). Efficacy of neurofeedback treatment in ADHD: The effects of inattention, impulsivity, and hyperactivity: A meta-analysis. Clinical EEG and Neuroscience, 40(3), 180-189. http://dx.doi.org/10.1177/155005940904000311

Fisher, M. A., Choudry, N. K., Brill, G., Avorn, J., Schneeweiss, S., Hutchins, D, ... Shrank, W. H. (2011). Trouble getting started: Predictors of primary medication nonadherence. American Journal of Medicine, 124, 1081. http://dx.doi.org/10.1016/j.amjmed.2011.05.028

Grencavage, L., \& Norcross, J. (1990). Where are the commonalities among the therapeutic common factors? Professional Psychology: Research and Practice, 21, 372-378. http://dx.doi.org/10.1037/0735-7028.21.5.372

Hammond, D. C. (2011). What is neurofeedback: An update. Journal of Neurotherapy, 15, 305-336. http://dx.doi.org/10.1080/10874208.2011.623090

Hammond, D. C., Bodenhamer-Davis, G., Gluck, G., Stokes, D., Hunt Harper, S., Trudeau, D., ... Kirk, L. (2011). Standards of practice for neurofeedback and neurotherapy: A position paper of the International Society for Neurofeedback and Research. Journal of Neurotherapy, 15, 54-64. http://dx.doi.org/10.1080/10874208.2010.545760

Hammond, D. C., \& Kirk, L. (2008). First, do no harm: Adverse effects and the need for practice standards in neurofeedback. Journal of Neurotherapy, 12, 79-88. http://dx.doi.org/10.1080/10874200802219947

Imel, Z., \& Wampold, B. (2008). The importance of treatment and the science of common factors in psychotherapy. In S. Brown \& R. Lent (Eds.), Handbook of counseling psychology (4th ed., pp. 249-262). Hoboken, NJ: John Wiley \& Sons Inc.

Larson, J. E. (In Press). Neurofeedback training increases practitioner profit. NeuroConnections.

Larson, J. E., Cothran, T., Drandorff, L., Morgan, C., \& Ryan, C. (2012). The relationship between burnout, interpersonal commitment, client adherence, and quality of work life among neurofeedback practitioners. Journal of Neurofeedback, 16(4), 237-245. http://dx.doi.org/10.1080/10874208.2012.728103

Larson, J. E., Ryan, C. B., \& Baerentzen, M. B. (2010). Practitioner perspectives of neurofeedback therapy for mental health and physiological disorders. Journal of Neurotherapy, 14, 280-290. http://dx.doi.org/10.1080/10874208.2010.523334

Lyle, R. R. (2012). Who we are, what do we do, and do we do it well? Journal of Neurotherapy, 16, 235-236. http://dx.doi.org/10.1080/10874208.2012.730433 
Mickey, R. M., Dunn, O. J., \& Clark, V. (2004). Applied statistics: An analysis of variance and regression (3rd ed.). Hoboken, NJ: John Wiley \& Sons, Inc.

Meisel, V., Servera, M., Garcia-Banda, G., Cardo, E., \& Moreno, I. (2013). Neurofeedback and standard pharmacological intervention in ADHD: A randomized controlled trial with six-month follow up. Biological Psychology, 94, 12-21. http://dx.doi.org/10.1016/j.biopsycho.2013.04.015

Niv, S. (2013). Clinical efficacy and potential mechanisms of neurofeedback. Personality and Individual Differences, 54, 676-686. http://dx.doi.org/10.1016/i.paid.2012.11.037

Sherlin, L. H., Arns, M., Lubar, J., Heinrich, H., Kerson, C., Strehl, U, \& Sterman, B. (2011). Neurofeedback and basic learning theory: Implications for research and practice. Journal of Neurotherapy, 15, 292-304. http://dx.doi.org/10.1080/10874208.2011.623089

Tracy, T. J. G., Lichtenberg, J. W., Goodyear, R. K., Claiborn, C. D., \& Wampold, B. E. (2003). Concept mapping of therapeutic common factors. Psychotherapy Research, 13, 401-413. http://dx.doi.org/10.1093/ptr/kpg041

Wampold, B. E., Mondin, G. W., Moody, M., Stich, F., Benson, K., \& Ahn, H. (1997). A metaanalysis of outcome studies comparing bona fide psychotherapies: Empirically, "All must have prizes." Psychological Bulletin, 122(3), 203-215. http://dx.doi.org/10.1037/0033-2909.122.3.203

Wogan, M., \& Norcross, J. C. (1985). Dimensions of therapeutic skills and techniques: Empirical identification, therapist correlates, and predictive utility. Psychotherapy: Theory, Research, Practice, Training, 22, 63-74. http://dx.doi.org/10.1037/h0088528

World Health Organization. (2003). Adherence to long-term therapies: Evidence for action. Geneva, Switzerland: Author.

Yucha, C., and Montgomery, D. (2008). Evidence-based practice in biofeedback and neurofeedback. Wheat Ridge, CO: Association for Psychophysiology and Biofeedback. 
Appendix A: NFB Practitioner Survey

1. What is your gender?

Male

Female

2. What is your age?

3. What is your highest level of education?

High School

Associate

Bachelor

Master

Doctorate

4. Are you a licensed mental health practitioner in your state?

Yes

No

5. Are you a licensed healthcare practitioner in your state?

Yes

No

6. How many years of neurofeedback experience do you have?

7. For an average month, how many hours of continuing education do you complete?

8. For an average month, how many neurofeedback sessions do you provide?

9. For an average month, how many clients do you have successfully completing their neurofeedback treatment?

10. For an average month, how many clients quit neurofeedback training before completing their neurofeedback treatment?

How would you rate your current knowledge about neurofeedback technology?

$\begin{array}{lllccc}\text { Poor } & \text { Fair } & \text { Good } & \text { Very Good } & \text { Excellent } \\ 1 & 2 & 3 & 4 & 5 & 6\end{array}$

How would you rate your current interpersonal skills with clients?

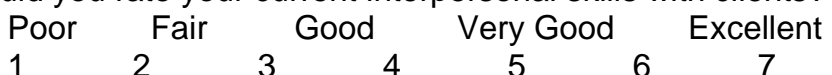

How would you rate your current commitment to learning about neurofeedback technology?

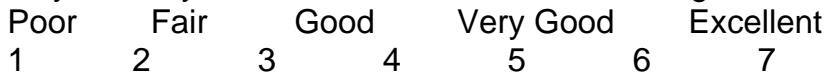

14. How would you rate your current commitment to improving your interpersonal skills with clients?

$\begin{array}{cccccc}\text { Poor } & \text { Fair } & \text { Good } & \text { Very Good } & \text { Excellent } \\ 1 & 2 & 3 & 4 & 5 & 6\end{array}$

15. My satisfaction level with my work life related to neurofeedback is?

0\% 10\% 20\% 30\% 40\% 50\% 60\% 70\% 80\% 90\% 100\%

16. My burnout level related to my neurofeedback practice is?

0\% 10\% 20\% 30\% 40\% 50\% 60\% 70\% 80\% 90\% 100\%

17. What is your frequency of doing neurofeedback training on yourself?

Not at all, Once a month, Once every other week, Once a week, Two times a week, Three times a week, Four times a week, Five times a week, Six times a week, Everyday

NOTE: Survey participants rated 12 traits for questions $18,19,20, \& 21$. Each question had 12 separate responses for a total of 48 items.

During a neurofeedback session, what is your satisfaction level with your ability to be...

(a) ethical, (b) attentive, (c) empathic, (d) calm, (e) observant, (f) humorous, (g) analytical, (h) confident, (i) friendly, (j) realistic, (k) optimistic, (I) careful

Very Dissatisfied Dissatisfied Neutral

$\begin{array}{llll}1 & 2 & 3 & 4\end{array}$

45

Very Satisfied

19. During a neurofeedback session, what is your priority level for being... 
(a) ethical, (b) attentive, (c) empathic, (d) calm, (e) observant, (f) humorous, (g) analytical, (h) confident, (i) friendly, (j) realistic, (k) optimistic, (I) careful

Not a priority

$\begin{array}{lcc}\text { Low } & \text { Somewhat } & \text { Neutral } \\ \text { Priority } & \text { Priority } & \text { Priority } \\ 2 & 3 & 4\end{array}$

Moderate
Priority
5
High
Priority
6
Essential
Priority
7

20. During a neurofeedback session, what is your level of difficulty or ease with being...

(a) ethical, (b) attentive, (c) empathic, (d) calm, (e) observant, (f) humorous, (g) analytical, (h) confident, (i) friendly, (j) realistic, (k) optimistic, (I) careful
Very Difficult
Somewhat
Neutral
Somewhat
Easy
$1 \quad 2$
3
4
Easy Very Easy
6
7

21. During a neurofeedback session, what is your satisfaction level with your ability to be...

(a) ethical, (b) attentive, (c) empathic, (d) calm, (e) observant, (f) humorous, (g) analytical, (h) confident, (i) friendly, (j) realistic, (k) optimistic, (I) careful

Not at all

1

2

3

Occasionally

$\begin{array}{lcccc}3 & 4 & 5 & 6 & 7\end{array}$




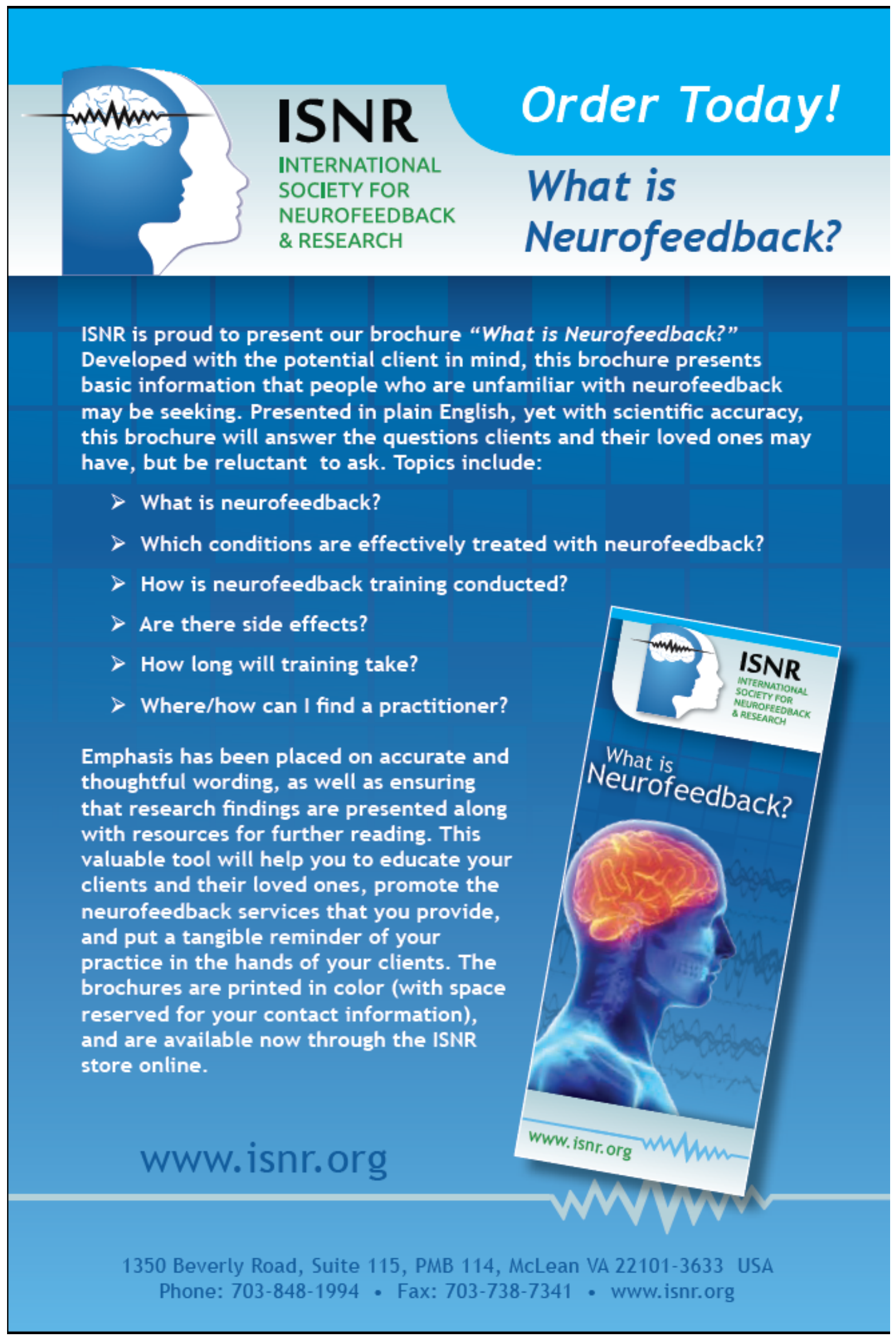

